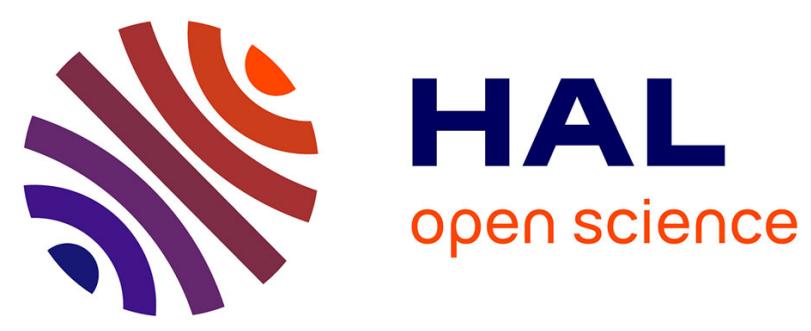

\title{
Multimodal assessment of sensorimotor shoulder function in patients with untreated anterior shoulder instability and asymptomatic handball players
}

Guillaume Mornieux, Anja Hirschmüller, Albert Gollhofer, Norbert P Südkamp, Dirk Maier

\section{To cite this version:}

Guillaume Mornieux, Anja Hirschmüller, Albert Gollhofer, Norbert P Südkamp, Dirk Maier. Multimodal assessment of sensorimotor shoulder function in patients with untreated anterior shoulder instability and asymptomatic handball players. Journal of Sports Medicine and Physical Fitness, 2018, 58 (4), pp.472-479. 10.23736/S0022-4707.17.06874-8 . hal-03102261

\section{HAL Id: hal-03102261 \\ https://hal.univ-lorraine.fr/hal-03102261}

Submitted on 23 Apr 2021

HAL is a multi-disciplinary open access archive for the deposit and dissemination of scientific research documents, whether they are published or not. The documents may come from teaching and research institutions in France or abroad, or from public or private research centers.
L'archive ouverte pluridisciplinaire HAL, est destinée au dépôt et à la diffusion de documents scientifiques de niveau recherche, publiés ou non, émanant des établissements d'enseignement et de recherche français ou étrangers, des laboratoires publics ou privés. 
Title: Multimodal assessment of sensorimotor shoulder function in patients with untreated anterior shoulder instability and asymptomatic handball players.

Authors: Guillaume Mornieux ${ }^{1^{*}}$, Anja Hirschmüller ${ }^{2}$, Albert Gollhofer ${ }^{3}$, Norbert P. Südkamp ${ }^{2}$ and Dirk Maier ${ }^{2}$

Faculty of Sport Sciences, University of Lorraine, EA $3450-$ DevAH, Nancy, France ${ }^{1}$

Department of Orthopaedics and Trauma Surgery, Medical Centre - Albert-LudwigsUniversity of Freiburg, Faculty of Medicine, Albert-Ludwigs-University of Freiburg, Germany $^{2}$

Institute for Sport and Sport Science, Albert-Ludwigs-University of Freiburg, Germany ${ }^{3}$

Corresponding author :

Guillaume Mornieux

University of Lorraine - Faculty of Sport Sciences - EA 3450 - DevAH

30 rue du Jardin Botanique - 54600 Villers-lès-Nancy, France

Tel.: +33 (0)3 83682931 - Fax: +33 (0)383682902

Email: guillaume.mornieux@ univ-lorraine.fr 


\section{Abstract}

Background. Functional evaluation of sensorimotor function of the shoulder joint is important for guidance of sports-specific training, prevention and rehabilitation of shoulder instability. Such assessment should be multimodal and comprise all qualities of sensorimotor shoulder function. This study evaluates feasibility of such multimodal assessment of glenohumeral sensorimotor function in patients with shoulder instability and handball players.

Methods. Nine patients with untreated anterior instability of their dominant shoulder and 15 asymptomatic recreational handball players performed proprioceptive joint position sense and dynamic stabilisation evaluations on an isokinetic device, as well as a functional throwing performance task. Outcome measures were analysed individually and equally weighted in a shoulder-specific sensorimotor index (S-SMI). Finally, isokinetic strength evaluations were conducted.

Results. We observed comparable sensorimotor functions of unstable dominant shoulders compared to healthy, contralateral shoulders (e.g. $\mathrm{P}=0.59$ for $\mathrm{S}-\mathrm{SMI}$ ). Handball players demonstrated superior sensorimotor function of their dominant shoulders exhibiting a significantly higher throwing performance and $\mathrm{S}-\mathrm{SMI}(\mathrm{P}<0.001$ and $\mathrm{P}=0.002$, respectively), but comparable internal rotator peak torques for both shoulders $(\mathrm{P}>0.22)$.

Conclusions. The present study proves feasibility of multimodal assessment of shoulder sensorimotor function in overhead athletes and patients with symptomatic anterior shoulder instability. Untreated shoulder instability led to a loss of dominance-related sensorimotor superiority indicating functional internal rotation deficiency. Dominant shoulders of handball players showed a superior overall sensorimotor function but weakness of dominant internal rotation constituting a risk factor for occurrence of posterior superior impingement syndrome. 
The S-SMI could serve as a diagnostic tool for guidance of sports-specific training, prevention and rehabilitation of shoulder instability.

Key words: glenohumeral instability, overhead sports, proprioception, joint position sense, perturbation, internal impingement, injury, prevention. 


\section{Introduction}

Evaluation of sensorimotor shoulder function is of major importance for guidance of sportsspecific training, injury prevention and rehabilitation of sports-specific shoulder disorders, e.g. shoulder instability and functional impingement syndromes.

The musculoskeletal sensorimotor system maintains functional joint stability by complex interaction of afferent, efferent, peripheral and central integration and processing components coordinating agonist and antagonist muscular activity. ${ }^{1}$ For instance, patients with anterior shoulder instability exhibit deficits of both afferent proprioception and efferent motor response. ${ }^{2}$ Mihata et al. showed that muscular imbalance and anterior capsular laxity lead to an increase of posterior superior glenohumeral contact pressures. ${ }^{3,4}$ Alterations of shoulder kinematics in anterior shoulder instability may lead to clinically symptomatic posterior superior impingement (PSI) syndrome causing structural lesions, e.g. superior labrum anterior to posterior (SLAP) lesions and tears of the posterior superior labrum as well as the rotator cuff. $^{3,5}$ PSI is known to be a common source of functional shoulder pain ("thrower's shoulder") in throwing athletes. ${ }^{6}$ Pathogenesis of the "thrower's shoulder" involves complex structural and kinematic alterations, most importantly a relative deficiency of internal rotation strength. Edouard et al. ${ }^{7}$ found muscular imbalances of internal and external rotation strength in asymptomatic, high-level handball players which were associated with a higher risk for subsequent shoulder injuries. Such muscular imbalance impairs sensorimotor shoulder function and may lead to clinically symptomatic functional disorders such as PSI and glenohumeral internal rotation deficit (GIRD) syndrome..$^{3,8,9}$

Proprioception plays an important role within the sensorimotor system. ${ }^{1}$ Subjects with unstable shoulders show decreased proprioceptive ability and mechanoreceptor dysfunction due to structural lesion of the capsuloligamentous complex. ${ }^{10}$ However, evaluation of 
proprioception alone, e.g., using joint position sense testing and detection of passive motion tests, ${ }^{11-13}$ does not provide sufficient information for comprehensive assessment of sensorimotor shoulder function because evaluation of efferent motor response is lacking. Literature reports shoulder range of motion tests ${ }^{14,15}$ and isokinetic strength evaluation in handball players ${ }^{7,16}$ and patients with anterior shoulder instability. ${ }^{17,18}$

However, few studies focused on sensorimotor function of the shoulder joint. ${ }^{19-23}$ While the latter focused on 3D analysis of upper extremity position reproduction during functional movement, others investigated glenohumeral sensorimotor control using an upper limb weight-bearing condition. Edouard et al. found impaired postural sensorimotor control in patients with anterior shoulder instability. ${ }^{19}$ The experimental set-up with the lower part of the body being supported on a table does not only target the shoulder joint but the entire upper body part and does not consider functional testing such as a throwing task.

To date, there is no experimental set-up that evaluates all relevant components of sensorimotor shoulder function including proprioception, dynamic neuromuscular control, muscle strength and functional performance.

The aim of the present study therefore was to evaluate feasibility and results of such multimodal assessment of sensorimotor shoulder function in patients with untreated anterior shoulder instability and asymptomatic recreational handball players. Both groups are prone to muscular imbalance and alteration of sensorimotor function potentially leading to functional shoulder disorders such as PSI and the "thrower's shoulder". Using combined proprioceptive, dynamic and functional testing, a shoulder-specific sensorimotor index (S-SMI) was designed as a novel diagnostic tool for multimodal assessment of sensorimotor shoulder function. We hypothesized 1) an impaired sensorimotor function of the dominant unstable shoulder compared to the non-dominant healthy shoulder in patients and 2) a superior sensorimotor 
function of the dominant throwing shoulder compared to the non-dominant shoulder in handball players.

\section{Materials and Methods}

Study population

Using data from the literature evaluating proprioceptive and isokinetic strength parameters in patients with shoulder instability ${ }^{12,17}$ and overhead athletes, ${ }^{13,16}$ the sample size was estimated to achieve $80 \%$ statistical power with an alpha level of 0.05 . Nine patients (PAT; 8 males and 1 female; body height $=180 \pm 7 \mathrm{~cm}$; body mass index $(\mathrm{BMI})=25 \pm 4 \mathrm{~kg} \cdot \mathrm{m}^{2}$; age $=22 \pm 4$ years) with untreated anterior instability exclusively of their dominant shoulders and 15 asymptomatic, recreational handball players (HB; 11 males and 4 females body height $=180$ $\pm 10 \mathrm{~cm} ; \mathrm{BMI}=24 \pm 3 \mathrm{~kg} \cdot \mathrm{m}^{2} ;$ age $=21 \pm 2$ years $)$ participated in the study. All patients had experienced at least one but a maximum of five shoulder dislocations with magnetic resonance imaging proven Bankart lesions of the anterior inferior labrum. Patients with bony involvement and associated injuries were excluded. The minimum time period between the last event of dislocation and multimodal testing was two months to ensure painless and full range of motion. None of the patients had received a specific rehabilitation program prior to testing. Patients suffered from subjective shoulder instability during overhead activities but must not have experienced habitual dislocations in daily routine. The clinical diagnosis of symptomatic, unidirectional anterior glenohumeral instability without ligamentous hyperlaxity (B2 according to Gerber and Nyfeller ${ }^{24}$ ) was confirmed by an experienced orthopaedic surgeon. All patients showed clearly positive anterior apprehension signs but no clinical signs of ligamentous hyperlaxity. Participants in the HB group were recruited to match the PAT group concerning body height, BMI and age. Handball players studying at the 
sport university and playing in the 3rd - 5th league, as well as their teammates were asked to participate in the study. They constituted a homogeneous group, with over 10 years handball experience and on average $6.5 \pm 1.5$ hours/week of training. The regular training program was maintained during testing. 13 players were right-handed and two were left-handed. All participants were free from any upper extremity pain at the time of testing and did not report any history of shoulder pain or injury over the 12 months prior to the measurements. In addition, players were excluded if they reported any history of shoulder dislocation, fracture or surgery.

Written consent was obtained prior to study participation. The study was approved by the local ethics committee (approval 195/12) and was conducted according to the ethical principles for medical research involving human subjects (Declaration of Helsinki).

\section{Procedure}

Participants were asked to perform proprioceptive joint position sense (JPS_prop) and dynamic stabilisation (Stabi_dyn) tests on an isokinetic device (IsoMed2000, D. \& R. Ferstl $\mathrm{GmbH}$, Hemau, Germany) in a seated position with $45^{\circ}$ of shoulder abduction in the scapular plane, $90^{\circ}$ of elbow flexion and $10^{\circ}$ of shoulder external rotation (Figure 1). Trunk and arm were firmly fixed with straps so that participants could not move these segments during measurements. The rationale for this testing position was the high reliability reported for isokinetic strength parameters. ${ }^{25}$ For JPS_prop testing, the shoulder was first placed in $10^{\circ}$ of internal rotation (IR) or $10^{\circ}$ of external rotation (ER). After $10 \mathrm{sec}$. the shoulder was turned to the neutral position. From that reference position, participants had to passively reposition their arm to the $10^{\circ}$ IR or ER positions, respectively. During the entire proprioception test, participants were blindfolded. 
For Stabi_dyn testing, participants had to maintain their shoulder position as close as possible to the reference position, while sustaining random displacements in both IR and ER directions. Intensity of displacements was set at different percentage levels of the maximal voluntary isometric torque (MVIT) measured in both IR and ER prior to testing. Additionally, instantaneous visual feedback of the arm position in relation to the reference position was provided on a screen. Two attempts at different levels of force (25\%, 50 and $75 \%$ MVIT) were allocated and each measurement lasted 54 seconds. Reliability of this new Stabi_dyn test was evaluated by means of the intraclass correlation coefficient $\left(\operatorname{ICC}_{2,1}\right)$. Therefore, among the 24 participants, 18 performed the test twice on 2 different days. Across the 3 intensity levels, this test showed acceptable reliability for internal and external rotations (ICC $=0.87$ and $\mathrm{ICC}=0.75$, respectively) ${ }^{26}$

In addition, participants conducted a functional throwing test to determine their individual performance index (FTPI_func) reflecting throwing accuracy. ${ }^{27}$ Subjects were asked to throw a ball towards a $0.3 \mathrm{~m}^{2}$ target area on the wall from a $4.6 \mathrm{~m}$ distance as many times as possible during a time period of 30 seconds. The examiner records the number of throws and the number of throws hitting the target area.

Finally, isokinetic strength testing was performed at 2 angular velocities: $90^{\circ} / \mathrm{s}$ and $180 \% \mathrm{~s}$ with 5 and 10 repetitions respectively. Participants were asked to progressively increase their effort in order to reach their peak strength during the last 2 repetitions.

All tests were performed for both the dominant (Do) and non-dominant shoulder (randomly assigned over all participants). Thus, the healthy non-dominant side (N-Do) provided intraindividual reference values for each participant. Few days prior to this multimodal assessment, participants became familiar with the testing procedure during a specific training session. 
Data analysis

Mean reposition errors $\left(\right.$ in $^{\circ}$ ) with respect of the $10^{\circ}$ IR or $10^{\circ}$ ER were assessed for JPS_prop testing. Mean position errors $\left(\right.$ in $^{\circ}$ ) related to the reference position were calculated over the 54 seconds test duration for IR and ER direction, respectively. FTPI_func (in \%) was calculated as the ratio between the number of accurate throws and the total number of throws during the 30 seconds test.

In order to further evaluate the sensorimotor function of the shoulder, a shoulder-specific sensorimotor index (S-SMI, expressed in \%) was calculated as follows:

$S-S M I(\%)=\frac{\overline{\text { JPS_prop }}+\overline{\text { Stabl_dyn }}+\overline{\text { FTPI_func }}}{3}$

Mean values $(\bar{x})$ of each of the three tests accounted for one third of the value of the S-SMI. For that purpose, all test results were expressed as percentages. For JPS_prop and Stabi_dyn measurements, 0 up to 10 degree deviation corresponded to percentage values ranging from $100 \%$ to $0 \%$, respectively.

During the isokinetic movements, dynamic strength of internal rotators and external rotators were evaluated by means of peak torque values normalized to body mass $\left(\mathrm{Nm}_{\mathrm{kg}}{ }^{-1}\right)$. Side-toside differences $(\%)$ were calculated at each angular velocity and represented dominant side strength deficit/superiority compared with non-dominant side.

Statistical analysis

The best out of two attempts of JPS_prop (two dependent variables), Stabi_dyn (six dependent variables) testing, FTPI_func, S-SMI and the peak torque values for IR and ER at 
each angular velocity were selected for both Do and N-Do shoulders for statistical analysis. Present data did not follow normal distribution for four variables (Shapiro-Wilk test). Accordingly, non-parametric tests were applied. Descriptive statistics used median, interquartile range as well as minimal and maximal values. The influence of shoulder dominance (Do vs. N-Do) on dependent variables within each participant group was analysed using the Wilcoxon test. The level of significance was set at 0.05 .

\section{Results}

PAT showed no significant difference between Do and N-Do shoulders for JPS_prop, Stabi_dyn and FTPI_func testing (Table I). Moreover, median (interquartile range) values for S-SMI were not significantly different between Do (44.7\% (15.5\%)) and N-Do (45.7\% (6.1\%)) shoulders $(\mathrm{P}=0.59)$.

HB achieved significantly superior Stabi_dyn scores for external rotation, at $25 \%$ level of force intensity for their Do compared to N-Do shoulders $(\mathrm{P}=0.004)$. FTPI_func was significantly higher for Do than $\mathrm{N}$-Do shoulder in handball players $(\mathrm{P}<0.001)$. Table II lists the detailed results of all variables for JPS_prop, Stabi_dyn and FTPI_func testing for HB. Moreover, S-SMI for Do $(56.9 \%(8.8 \%))$ was significantly higher than for N-Do $(45.4 \%$ $(8.2 \%)$ ) shoulders $(\mathrm{P}=0.002$; Figure 2$)$.

Internal and external rotator isokinetic peak torque values and external to internal rotator ratios (ER/IR) values for PAT and $\mathrm{HB}$ are presented in Table III. Moreover, side-to-side differences (Do vs. N-Do) for these values are provided in Table III. IR values were not significantly different between Do and N-Do. For instance, HB demonstrated comparable IR values between Do and $\mathrm{N}-\mathrm{Do}$ at $90 \% \mathrm{sec}$ and $180 \% \mathrm{sec}(\mathrm{P}=0.59$ and $\mathrm{P}=0.22$, respectively). ER for Do was significantly higher than for N-Do shoulders for both PAT $(\mathrm{P}=0.01)$ and HB 
$(\mathrm{P}=0.005)$ at $90 \%$ sec. The same was true at $180^{\circ} / \mathrm{sec}(\mathrm{P}=0.012$ and $\mathrm{P}=0.02$, respectively). ER/IR ratios were also significantly higher for Do than for N-Do shoulders for both PAT $(\mathrm{P}=0.02)$ and $\mathrm{HB}(\mathrm{P}=0.03)$ at $90^{\circ} / \mathrm{sec}$. At $180 \% \mathrm{sec}, \mathrm{ER} / \mathrm{IR}$ ratio was significantly higher for Do than for N-Do shoulders for PAT $(\mathrm{P}=0.01)$, but not HB $(\mathrm{P}=0.05)$.

\section{Discussion}

Multimodal assessment of the glenohumeral sensorimotor function revealed 1) successful and valid measurements in both patients with shoulder instability and handball players, 2) comparable sensorimotor function of unstable dominant shoulders compared to healthy contralateral shoulders and 3) superior sensorimotor function of the dominant shoulder of handball players compared to their non-dominant side.

Neither singular sensorimotor tests nor S-SMI were significantly influenced by glenohumeral instability of dominant shoulders. Edouard et al. ${ }^{19}$ found a significant sensorimotor control deficiency of $20-25 \%$ in patients with unstable dominant shoulders compared to healthy contralateral shoulders. However, they employed a static, weight-bearing experimental set-up, that rather measured postural stability of both the upper body than glenohumeral sensorimotor function. To our knowledge, the present study is the first to assess the influence of shoulder stability on dynamic sensorimotor shoulder function and adds valuable information to exclusive muscular strength analysis. Numerous studies showed significant isokinetic strength differences between unstable and stable shoulders. ${ }^{17,18,28}$ However, only Edouard et al. ${ }^{17}$ investigated rotator cuff strength profile in glenohumeral instability, while ensuring that instability was only present on the dominant side, as we did in the present study. These authors found a significant reduction of up to $9 \%$ of isokinetic peak torque values for external rotation but no significant difference for internal rotation in 
unstable compared to contralateral stable shoulders. ${ }^{17}$ The lack of internal rotation difference between both shoulders has also been reported in the present study. On the other hand, the literature consistently reports significant higher internal (up to 15\%) and external (up to 12\%) rotation strength of healthy dominant compared to healthy non-dominant shoulders. ${ }^{7,17,29}$

Present findings cannot be directly compared with previously published studies. Most importantly, there exist no reference data for dynamic stabilisation testing with perturbation. In contrast, sensorimotor shoulder function was assessed using a novel dynamic perturbation model in the present study. Based on existent knowledge, one would assume significant superiority of about $15 \%$ of sensorimotor function of the dominant shoulder joint. In the present study, untreated anterior shoulder instability led to a loss of this dominance-related effect indicating a significant decrease of sensorimotor function.

Handball players demonstrated superior dynamic stabilization of their dominant shoulder joints for external rotation at the lowest level of force intensity (25\% MVIT), a higher functional throwing ability and an increased S-SMI compared to the non-dominant side.

Superior sensorimotor control for external rotation at the lowest force level might be explained by intensive functional training of dominant, throwing shoulders. However, this effect was not consistently present and could not be detected for higher force levels and internal rotation. This new dynamic stabilisation test combines proprioceptive and functional testing, is reliable and can be performed at individual force levels. Different experimental settings (e.g., lower force levels) should be explored to detect instability-related and sportsspecific deficits and imbalances. This comprehensive sensorimotor evaluation showed comparable internal rotator peak torques for both shoulders, and adds substantial knowledge to findings from conventional isokinetic strength studies. In contrast, other studies on elite handball players observed significantly higher isokinetic internal rotation strengths for 
dominant, throwing shoulders. ${ }^{7,30-32}$ We explained this discrepancy by the lower level of training intensity and competition of recreational compared to high-level professional athletes.

The functional throwing test showed that dominant shoulders of handball players reached a $60 \%$ FTPI value corresponding to the top of the range reported for healthy shoulders. ${ }^{27}$ Moreover, non-dominant shoulders as well as unstable shoulders reached a poor FTPI level of below 30\%. These findings indicate that instability-caused impairment of shoulder sensorimotor function might be effectively detected by functional tasks such as throwing, which are performed at low force levels.

Increased functional throwing ability combined with superior dynamic stabilisation for external rotation at a low force level yielded a superior S-SMI for dominant compared to nondominant shoulders of handball players.

Passive joint position sense was neither improved in dominant shoulders of handball players nor reduced in unstable dominant shoulders compared to non-dominant healthy shoulders. Although patients showed a $2^{\circ}$ less accurate unstable shoulder for internal rotation, this value did not reach statistical significance. Our results were in accordance with joint position sense values found by Lephart et al. ${ }^{12}$ Consistently, Safran et al. ${ }^{13}$ reported symmetry of shoulder proprioception in overhead athletes. Our study did not detect side-specific differences for shoulder proprioception using joint position sense testing, while combined functional and dynamic testing (FTPI_func and Stabi_dyn) enabled comprehensive evaluation of sensorimotor function of the shoulder joint.

This study represents a novel approach of comprehensive multimodal evaluation of sensorimotor function of the shoulder joint comprising sensory, motor and functional qualities. The use of an isokinetic dynamometer to perform dynamic stabilisation tests further 
shows the considerable potential of this technology. But several limitations need to be considered. Most importantly, normative values of sensorimotor function need to be defined by assessment of representative control cohorts. Dynamic sensorimotor testing at lower force levels might enable the detection of significant differences and the inclusion of patients with higher grades of shoulder instability.

From a clinical perspective, the combination of relative internal rotation weakness and anterior capsular laxity in untreated shoulder instability entails a substantial risk for posterior superior glenohumeral impingement and subsequent structural lesions, e.g. articular-sided rotator cuff and labral tears. ${ }^{3-5,33}$ Interestingly, this was also true for dominant shoulders of recreational handball players. Overhead athletes such as handball players are prone to symptomatic posterior superior impingement due to a relative weakness of internal rotation strength and glenohumeral internal rotation deficit (GIRD)., ${ }^{3,5,6,8}$ Though, all included handball players were clinically asymptomatic, dominant internal rotation weakness represents a risk factor for occurrence of symptomatic posterior superior impingement syndrome. In this context, specific training of internal rotation strength appears beneficial for both patients with untreated shoulder instability and recreational handball players performing at lower levels of competition.

\section{Conclusions}

The present study proves feasibility of multimodal assessment of shoulder sensorimotor function in high-demand overhead athletes, healthy individuals and patients with symptomatic anterior shoulder instability. Untreated shoulder instability led to a loss of dominance-related superiority indicating functional internal rotation strength deficiency. Dominant shoulders of handball players showed a superior overall sensorimotor function but weakness of dominant 
internal rotation constituting a risk factor for occurrence of posterior superior impingement syndrome. The S-SMI, including proprioception, dynamic stabilisation and functional throwing ability, may serve as a diagnostic tool for guidance of sports-specific training, injury prevention and rehabilitation of shoulder instability and possibly other sports-specific shoulder disorders. Internal rotation training appears beneficial for both patients with untreated anterior shoulder instability and handball players performing at a recreational level.

\section{Acknowledgment and Conflict of Interest}

Each author has participated to the study. The authors state that the article is original, has not been submitted elsewhere and that the study was approved by the ethics committee. No potential conflict of interest was reported by the authors. 


\section{References}

1. Riemann BL, Lephart SM. The sensorimotor system, part I: the physiologic basis of functional joint stability. J Athl Train 2002;37:71-9.

2. Myers JB, Wassinger CA, Lephart SM. Sensorimotor contribution to shoulder stability: effect of injury and rehabilitation. Man Ther 2006;11:197-201.

3. Mihata T, Gates J, Mcgarry MH, Lee J, Kinoshita M, Lee TQ. Effect of rotator cuff muscle imbalance on forceful internal impingement and peel-back of the superior labrum: a cadaveric study. Am J Sports Med 2009;37:2222-7.

4. Mihata T, Mcgarry MH, Neo M, Ohue M, Lee TQ. Effect of anterior capsular laxity on horizontal abduction and forceful internal impingement in a cadaveric model of the throwing shoulder. Am J Sports Med 2015;43:1758-63.

5. Walch G, Boileau P, Noel E, Donell ST. Impingement of the deep surface of the supraspinatus tendon on the posterosuperior glenoid rim: an arthroscopic study. J Shoulder Elbow Surg 1992;1:238-45.

6. Liu SH, Boynton E. Posterior superior impingement of the rotator cuff on the glenoid rim as a cause of shoulder pain in the overhead athlete. Arthroscopy 1993;9:697-9.

7. Edouard P, Degache F, Oullion R, Plessis JY, Gleizes-Cervera S, Calmels P. Shoulder strength imbalances as injury risk in handball. Int J Sports Med 2013;34:654-60.

8. Guney H, Harput G, Colakoglu F, Baltaci G. The effect of glenohumeral internal-rotation deficit on functional rotator-strength ratio in adolescent overhead athletes. J Sport 
Rehabil 2016;25:52-7.

9. Myers J, Lephart S. The role of the sensorimotor system in the athletic shoulder. J Athl Training35:351-63;2000 .

10. Warner JJP, Lephart S, Fu FH. Role of proprioception in pathoetiology of shoulder instability. Clin Orthop Relat Res 1996;330:35-9.

11. Aydin T, Yildiz Y, Yanmis I, Yildiz C, Kalyon TA. Shoulder proprioception: a comparison between the shoulder joint in healthy and surgically repaired shoulders. Arch Orthop Trauma Surg 2001;121:422-5.

12. Lephart SM, Warner JJP, Borsa PA, Fu FH. Proprioception of the shoulder joint in healthy, unstable, and surgically repaired shoulders. J Shoulder Elbow Surg 1994;3:37180.

13. Safran MR, Borsa PA, Lephart SM, Fu FH, Warner JJP. Shoulder proprioception in baseball pitchers. J Shoulder Elbow Surg 2001;10:438-44.

14. Wilk KE, Meister K, Andrews JR. Current concepts in the rehabilitation of the overhead throwing athlete. Am J Sports Med 2002;30:136-51.

15. Zheng N, Eaton K. Shoulder rotational properties of throwing athletes. Int J Sports Med $2012 ; 33: 463-8$.

16. Andrade MDS, Fleury AM, de Lira CAB, Dubas JP, da Silva AC. Profile of isokinetic eccentric-to-concentric strength ratios of shoulder rotator muscles in elite female team handball players. J Sports Sci 2010;28:743-9. 
17. Edouard P, Degache F, Beguin L, Samozino P, Gresta G, Fayolle-Minon I et al. Rotator cuff strength in recurrent anterior shoulder instability. J Bone Joint Surg Am 2011;93:759-65.

18. Saccol MF, Zanca GG, Ejnisman B, de Mello MT, Mattiello SM. Shoulder rotator strength and torque steadiness in athletes with anterior shoulder instability or SLAP lesion. J Sci Med Sport 2014;17:463-68.

19. Edouard P, Gasq D, Calmels P, Degache F. Sensorimotor control deficiency in recurrent anterior shoulder instability assessed with a stabilometric force platform. J Shoulder Elbow Surg 2014;23:355-60.

20. Laudner KG. Upper extremity sensorimotor control among collegiate football players. J Strength Cond Res 2012;26:672-6.

21. Myers JB, Guskiewicz KM, Schneider RA, Prentice WE. Proprioception and neuromuscular control of the shoulder after muscle fatigue. J Athl Train 1999;34:362-7.

22. Padua DA, Guskiewicz KM, Prentice WE, Schneider RE, Shields EW. The effect of select shoulder exercises on strength, active angle reproduction, single-arm balance, and functional performance. J Sport Rehabil 2004;13:94-114.

23. Tripp BL, Yochem EM, Uhl TL. Recovery of upper extremity sensorimotor system acuity in baseball athletes after a throwing-fatigue protocol. J Athl Train 2007;42:90-8.

24. Gerber C, Nyffeler RW. Classification of glenohumeral joint instability. Clin Orthop Relat Res 2002;400:65-76. 
25. Edouard P, Samozino P, Julia M, Gleizes-Cervera S, Vanbiervliet W, Calmels P et al. Reliability of isokinetic assessment of shoulder-rotator strength: a systematic review of the effect of position. J Sport Rehabil 2011;20:367-83.

26. Hopkins WG. Measures of reliability in sports medicine and science. Sports Med $2000 ; 30: 1-15$.

27. Davies GJ, Dickoff-hoffman S. Neuromuscular testing and rehabilitation of the shoulder complex. J Orthop Sports Phys Ther 1993;18:449-58.

28. Tsai L, Wredmark T, Johansson C, Gibo K, Engstrom B, Tornqvist H. Shoulder function in patients with unoperated anterior shoulder instability. Am J Sports Med 1991;19:46973.

29. Mayer F, Horstmann T, Röcker K, Heitkamp HC, Dickhuth HH. Normal values of isokinetic maximum strength, the strength/velocity curve, and the angle at peak torque of all degrees of freedom in the shoulder. Int J Sports Med 1994;15 Suppl 1:S19-25.

30. Clarsen B, Bahr R, Andersson SH, Munk R, Myklebust G. Reduced glenohumeral rotation, external rotation weakness and scapular dyskinesis are risk factors for shoulder injuries among elite male handball players: a prospective cohort study. Br J Sports Med 2014;48:1327-33.

31. Andrade MDS, de Lira CAB, Vancini RL, de Almeida AA, Benedito-Silva AA, da Silva AC. Profiling the isokinetic shoulder rotator muscle strength in 13- to 36-year-old male and female handball players. Phys Ther Sport 2013;14:246-52.

32. Ellenbecker TS, Davies GJ. The application of isokinetics in testing and rehabilitation of 
the shoulder complex. J Athl Train 2000;35:338-50.

33. Warner JJP, Micheli LJ, Arslanian LE, Kennedy J, Kennedy R. Patterns of flexibility, laxity, and strength in normal shoulders and shoulders with instability and impingement. Am J Sports Med 1990;18:366-75. 


\section{Tables and Figures captions}

Table I: Median, interquartile range (IQR), minimal and maximal values of proprioception (JPS_prop) and dynamic stabilisation (Stabi_dyn) scores for internal (IR) and external shoulder rotation (ER) and the three force levels $(25,50,75 \%)$, as well as functional throwing (FTPI_func) scores for the dominant unstable (Do) and non-dominant (N-Do) shoulders in the patients group.

Table II: Median, interquartile range (IQR), minimal and maximal values of proprioception (JPS_prop) and dynamic stabilisation (Stabi_dyn) scores for internal (IR) and external shoulder rotation (ER) and the three force levels $(25,50,75 \%)$, as well as functional throwing (FTPI_func) scores for the dominant (Do) and non-dominant (N-Do) shoulders in the handball players group.

*indicates significant differences between Do and N-Do.

Table III: Median (interquartile range) values for internal rotator and external rotator peak torque and external to internal rotator ratios (ER/IR) for patients with anterior instability and handball players.

* indicates significant differences between Do and N-Do.

Figure 1: Schematic view of the setup during the joint position sense and sensorimotor tests on the isokinetic device. 
Figure 2: Boxplot of the differences in the shoulder-specific sensorimotor index between dominant (Do) and non-dominant (N-Do) shoulders for both patients and handball players. Median, first and third quartiles as well as whiskers from minimum to maximum are depicted on the figure.

** indicates significant differences $(\mathrm{P}<0.01)$. 
Table I: Differences in glenohumeral sensorimotor function between dominant and non-dominant shoulders for patients with anterior instability.

\begin{tabular}{|c|c|c|c|c|c|c|c|c|c|}
\hline & \multicolumn{4}{|c|}{ Patients Do } & \multicolumn{4}{|c|}{ Patients N-Do } & \multirow{2}{*}{$\begin{array}{c}\text { Shoulder } \\
\text { effect } \\
\text { P value }\end{array}$} \\
\hline & Median & IQR & Min & Max & Median & IQR & Min & Max & \\
\hline JPS_prop IR $\left(^{\circ}\right)$ & 4 & 1 & 0 & 6 & 2 & 2 & 0 & 7 & 0.67 \\
\hline JPS_prop ER $\left(^{\circ}\right)$ & 5 & 5 & 2 & 15 & 6 & 3 & 0 & 13 & 0.73 \\
\hline Stabi_dyn IR 25\% $\left(^{\circ}\right)$ & 2.5 & 0.9 & 2.1 & 3.8 & 3.2 & 0.9 & 1.9 & 4.1 & 0.17 \\
\hline Stabi_dyn ER $25 \%\left(^{\circ}\right)$ & 2.8 & 0.8 & 1.8 & 5.1 & 3.9 & 1.8 & 2.4 & 5.3 & 0.21 \\
\hline Stabi_dyn IR 50\% $\left(^{\circ}\right)$ & 4.5 & 0.1 & 3 & 5.9 & 4.5 & 1.7 & 3.3 & 6.2 & 0.62 \\
\hline Stabi_dyn ER 50\% $\left(^{\circ}\right)$ & 3.7 & 2.4 & 2.7 & 7.5 & 4.8 & 0.6 & 3.8 & 8.3 & 0.11 \\
\hline Stabi_dyn IR 75\% $\left(^{\circ}\right)$ & 6 & 1.1 & 4.3 & 10.1 & 5.9 & 2.1 & 4.1 & 11.6 & 0.94 \\
\hline Stabi_dyn ER 75\% $\left(^{\circ}\right)$ & 6 & 3.4 & 4.1 & 13.3 & 7.2 & 3.4 & 5.6 & 11.8 & 0.37 \\
\hline FTPI_func (\%) & 27.3 & 13.5 & 20 & 50 & 28.6 & 5.8 & 0 & 66.7 & 0.77 \\
\hline
\end{tabular}


Median, interquartile range (IQR), minimal and maximal values of proprioception (JPS_prop) and dynamic stabilisation (Stabi_dyn) scores for internal (IR) and external shoulder rotation (ER) and the three force levels $(25,50,75 \%)$, as well as functional throwing (FTPI_func) scores for the dominant unstable (Do) and non-dominant (N-Do) shoulders in the patients group. 
Table II: Differences in glenohumeral sensorimotor function between dominant and non-dominant shoulders for handball players.

\begin{tabular}{|c|c|c|c|c|c|c|c|c|c|}
\hline & \multicolumn{4}{|c|}{ Handball Players Do } & \multicolumn{4}{|c|}{ Handball Players N-Do } & \multirow{2}{*}{$\begin{array}{c}\text { Shoulder } \\
\text { effect } \\
\text { P value }\end{array}$} \\
\hline & Median & IQR & Min & Max & Median & IQR & Min & $\operatorname{Max}$ & \\
\hline JPS_prop IR $\left(^{\circ}\right)$ & 3 & 2 & 0 & 8 & 2 & 5 & 0 & 10 & 0.62 \\
\hline JPS_prop ER $\left(^{\circ}\right)$ & 5 & 5 & 2 & 11 & 6 & 7 & 1 & 11 & 0.89 \\
\hline Stabi_dyn IR 25\% $\left(^{\circ}\right)$ & 2.6 & 0.7 & 1.8 & 3.4 & 2.9 & 0.7 & 1.9 & 3.3 & 0.36 \\
\hline Stabi_dyn ER 25\% $\left(^{\circ}\right)$ & 3.1 & 0.6 & 2.3 & 3.9 & 3.7 & 0.7 & 2.7 & 5.9 & $0.004 *$ \\
\hline Stabi_dyn IR 50\% $\left(^{\circ}\right)$ & 4 & 0.8 & 3.3 & 4.9 & 4.3 & 0.8 & 2.9 & 5.1 & 0.15 \\
\hline Stabi_dyn ER 50\% $\left(^{\circ}\right)$ & 4.5 & 1.2 & 3.1 & 6 & 4.8 & 1.3 & 3.3 & 6.1 & 0.17 \\
\hline Stabi_dyn IR 75\% $\left(^{\circ}\right)$ & 5.4 & 1.7 & 4.4 & 7.1 & 5.7 & 0.9 & 4.2 & 8.6 & 0.51 \\
\hline Stabi_dyn ER 75\% $\left(^{\circ}\right)$ & 5.8 & 1.4 & 3.9 & 8.8 & 6 & 1.3 & 4.3 & 10.5 & 0.33 \\
\hline FTPI_func (\%) & 60 & 10.4 & 43.8 & 85.7 & 31.3 & 13.5 & 9.1 & 46.2 & $<0.001 *$ \\
\hline
\end{tabular}


Median, interquartile range (IQR), minimal and maximal values of proprioception (JPS_prop) and dynamic stabilisation (Stabi_dyn) scores for internal (IR) and external shoulder rotation (ER) and the three force levels $(25,50,75 \%)$, as well as functional throwing (FTPI_func) scores for the dominant (Do) and non-dominant (N-Do) shoulders in the handball players group.

*indicates significant differences between Do and N-Do. 
Table III: Median (interquartile range) values for internal rotator and external rotator peak torque and external to internal rotator ratios (ER/IR)

for patients with anterior instability and handball players

\begin{tabular}{|c|c|c|c|c|c|c|}
\hline & \multicolumn{3}{|c|}{ Patients } & \multicolumn{3}{|c|}{ Handball Players } \\
\hline & Do & N-Do & Do vs. N-Do (\%) & Do & N-Do & Do vs. N-Do (\%) \\
\hline \multicolumn{7}{|c|}{ Isokinetic $90^{\circ} / \mathrm{sec}(\mathrm{Nm} / \mathrm{kg})$} \\
\hline External Rotators & $0.37(0.07)$ & $0.29(0.11) *$ & $28.6(30.0)$ & $0.41(0.15)$ & $0.33(0.15) *$ & $46.9(35.2)$ \\
\hline ER/IR ratio & $0.82(0.43)$ & $0.57(0.12) *$ & $62.5(40.8)$ & $0.72(0.43)$ & $0.47(0.28) *$ & $58.0(63.2)$ \\
\hline \multicolumn{7}{|c|}{ Isokinetic $180^{\circ} / \mathrm{sec}(\mathrm{Nm} / \mathrm{kg})$} \\
\hline Internal Rotators & $0.50(0.17)$ & $0.58(0.16)$ & $-14.3(17.2)$ & $0.61(0.25)$ & $0.66(0.22)$ & $-5.1(13.4)$ \\
\hline External Rotators & $0.40(0.10)$ & $0.30(0.17) *$ & $43.5(65.9)$ & $0.45(0.20)$ & $0.34(0.18) *$ & $40(35.7)$ \\
\hline ER/IR ratio & $0.86(0.35)$ & $0.53(0.17) *$ & $91.3(75.9)$ & $0.72(0.37)$ & $0.49(0.38)$ & $53.4(53.2)$ \\
\hline
\end{tabular}

The dominant side in the patients group corresponds to the unstable shoulder.

*indicates significant differences between Do and N-Do. 


$$
y
$$




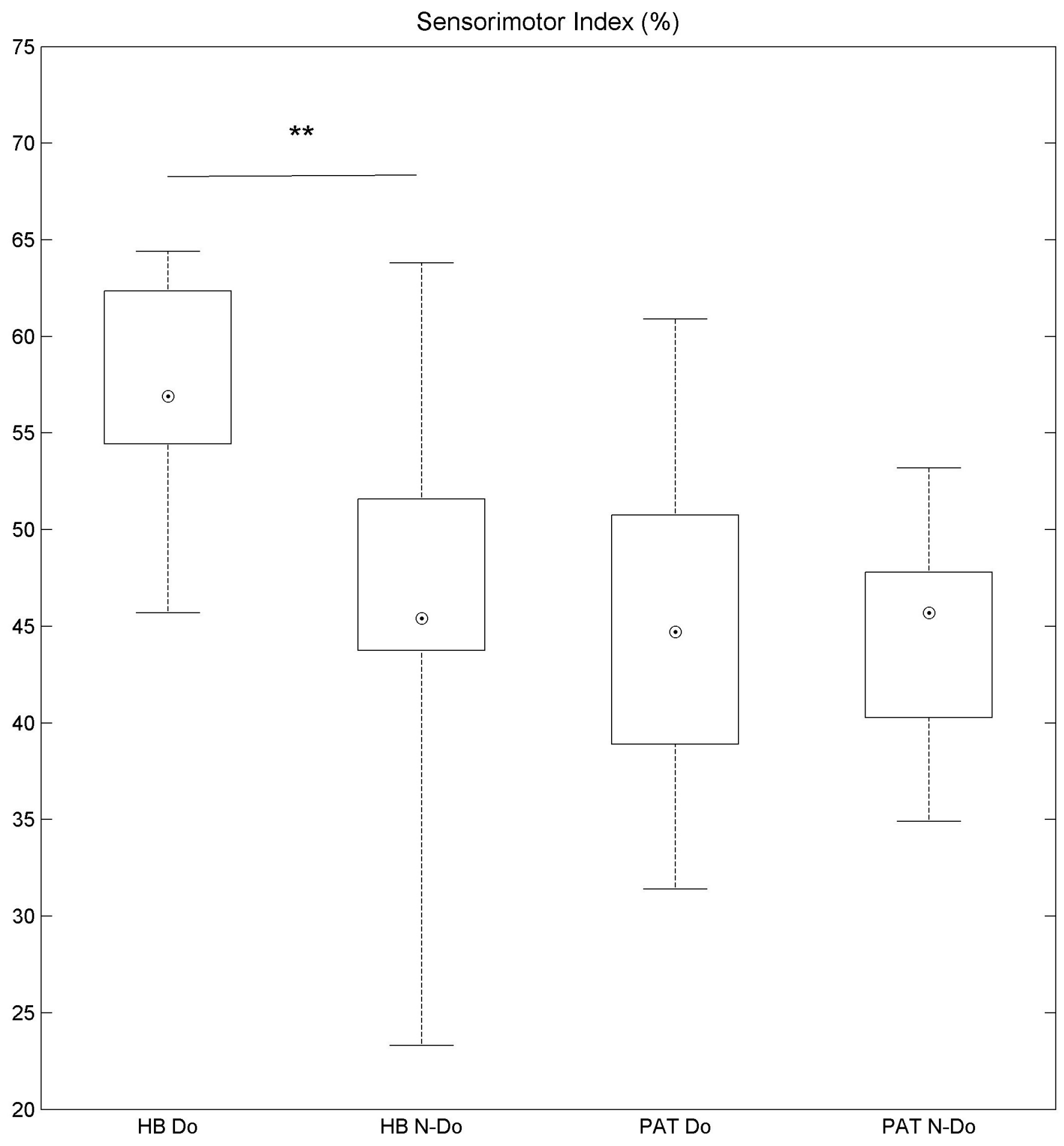

